\title{
A comparison of methods for staining tubercle bacilli in histological sections
}

\author{
N. GREENWOOD AND H. FOX \\ From the Department of Pathology, University of Manchester
}

SYNOPSIS The Ziehl-Neelsen stain is compared with three alternative methods of staining tubercle bacilli in paraffin sections: Fite's method (1938); a modification by Armstrong and Price (1947) of Fite's method; and a fluorescent method using the auramine-phenol stain.

The fluorescent method was found to be the most effective of the four methods and its use is recommended as a routine tool in diagnostic histopathology.

The Ziehl-Neelsen stain is the most commonly used technique in Britain for the demonstration of tubercle bacilli in histological sections. In our experience, however, this stain yields a disquietingly low incidence of positive results in sections from lesions which are histologically typical of tuberculosis. In such cases, a diagnosis of tuberculosis is often made in spite of the failure to demonstrate the organism, although a slight doubt as to the true nature of the lesion may remain in the pathologist's mind.

Any technique which produces better results than those obtained with the Ziehl-Neelsen stain would therefore be welcome to histologists. This investigation involves a comparison of the Ziehl-Neelsen stain with two alternative fuchsin stains and with a fluorescent technique in an attempt to determine which is of the most value in the routine examination of histological sections.

\section{Material}

Seventy cases which had been diagnosed as tuberculosis' were selected at random from the files of the Department of Pathology, University of Manchester. Sections from all these cases showed epithelioid cell granulomata and areas of caseation. The tissues were from a variety of anatomical sites including lymph nodes (21), kidney (9), gastrointestinal tract (6), epididymus (5), liver (4), omentum (4), synovium (3), brain (1), vagina (1), heart (1), spleen (1), and skin (1).

Sections from each case were cut and stained by the following methods:

Received for publication 3 October 1972.

\section{Ziehl-Neelsen Method}

METHOD

1 Bring sections to water.

2 Stain in hot carbol-fuchsin in a coplin jar at $50^{\circ} \mathrm{C}$ for 30 minutes.

3 Wash in water to remove excess stain.

4 Differentiate in $3 \% \mathrm{HCl}$ in $70 \%$ alcohol until pale pink for five to 10 minutes.

5 Wash in water.

6 Counterstain in $0.1 \%$ methylene blue for 10 to 15 seconds.

7 Wash in water.

8 Dehydrate, clear, and mount.

RESULTS

Tubercle bacilli stain red.

\section{Fite's Method}

Fite $(1938,1940)$ introduced a new fuchsinformaldehyde stain for lepra bacilli, which was thought to be also useful for the demonstration of tubercle bacilli in histological material.

\section{METHOD}

1 Bring sections to water.

2 Stain in new fuchsin $0.5 \mathrm{~g}$, phenol crystals $5.0 \mathrm{~g}$, ethanol $10 \mathrm{ml}$, and distilled water to make $100 \mathrm{ml}-$ at $60^{\circ} \mathrm{C}$ (paraffin oven) for 12 to 24 hours.

3 Reagent grade, $40 \%$ formaldehyde for five minutes. 4 Hydrochloric acid $2 \%$ in $95 \%$ alcohol for 10 minutes.

5 Potassium permanganate $1 \%$ aqueous for two to five minutes. 
6 Oxalic acid $2 \%$ for one minute.

7 Harris' haematoxylin for two minutes.

8 Van Gieson's stain for three minutes.

9 Dehydrate, clear, and mount.

Each of the above steps is preceded by washing in water.

RESULTS

Tubercle bacilli stain blue.

\section{Armstrong Price Method}

Fite's method was later modified and shortened by Armstrong and Price (1947).

\section{METHOD}

1 Bring the sections to water.

2 Stain in new fuchsin $0.5 \mathrm{~g}$, phenol crystals $5.0 \mathrm{~g}$, ethanol $10 \mathrm{ml}$, and distilled water to make $100 \mathrm{ml}$. 3 Reagent grade, $40 \%$ formaldehyde for five minutes.

4 Hydrochloric acid $1 \%$ in $70 \%$ alcohol for five minutes.

5 Potassium permanganate $1 \%$ aqueous for two to five minutes.

6 Oxalic acid $2 \%$ for 30 seconds.

7 Harris' haematoxylin for two minutes.

8 Van Gieson stain for three minutes.

9 Dehydrate, clear, and mount.

Each of the above steps is preceded by washing in water.

RESULTS

Tubercle bacilli stain blue.

\section{Auramine Phenol Fluorescent Method}

Mansfield (1970) compared 14 different fluorescent methods for the detection of tubercle bacilli in histological sections and found the auramine phenol stain to be the most satisfactory.

\section{REAGENTS}

The xylene-oil deparaffinizer solution is made up of xylene 2 parts and arachis oil 1 part.

\section{Auramine O-phenol stain}

$1 \mathrm{Mix} 40 \mathrm{ml}$ phenol and $60 \mathrm{ml}$ glycerine in a graduated container.

2 Pour phenol-glycerine solution over $3.0 \mathrm{~g}$ auramine $O$ in a litre flask; mix for five minutes. 3 Add $900 \mathrm{ml}$ distilled water, rinsing the phenolglycerine container at least three times. Cover the mouth of the flask with Parafilm to prevent evaporation. Mix the solution for 72 hours using a magnetic stirrer.
4 Set the mixed solution in the dark for four days then filter through Millipore AP 20 prefilters, changing the filter pads frequently.

5 Store in brown bottles.

Acid-alcohol decolourizing solution

Concentrated hydrochloric acid, $0.5 \mathrm{ml}$, and $70 \%$ ethanol $99.5 \mathrm{ml}$.

\section{Potassium permanganate counterstain}

Potassium permanganate $0.5 \mathrm{~g}$ per $100 \mathrm{ml}$ distilled water. Store in a dark bottle.

\section{METHOD}

1 Deparaffinize sections with xylene-oil mixture two changes, six minutes each.

2 Rinse in running tap water for one minute Drain slides for 45 seconds, but do not remove all the oil.

3 Stain with phenol-auramine $\mathrm{O}$ stain for 15 minutes at $25-30^{\circ} \mathrm{C}$.

4 Rinse in running tap water.

5 Decolourize in $0.5 \%$ acid-alcohol for one minute. 6 Counterstain slides with $0.5 \%$ potassium permanganate solution for three minutes.

7 Rinse in running tap water.

8 Air dry with drainage.

9 Wipe off excess stain and oil around the sections. 10 Mount in low fluorescing mounting medium (Gurr's Univert mountant).

\section{RESULTS}

Tubercle bacilli fluoresce white against a black background (figs 1, 2, and 3 ).

\section{Controls}

Positive controls were included with each batch of sections stained by all the above methods. In addition, negative controls were carried out with the fluorescent method, using various tissues from patients with Crohn's disease, sarcoidosis, foreign body granulomata, and including paraffin wax controls to rule out contamination during processing.

\section{Microscopy}

All sections were examined initially using a $\times 40$ objective and $\times 10$ eyepieces; they were then reexamined using $\times 100$ oil-immersion objective and $\times 10$ eyepieces. Sections stained by the fluorescent method were also examined using a $\times 25$ objective and $\times 10$ eyepieces. Sections stained by the fluorescent method were examined under a fluorescent microscope with appropriate heat and barrier filters and BG12 excitation filter. 


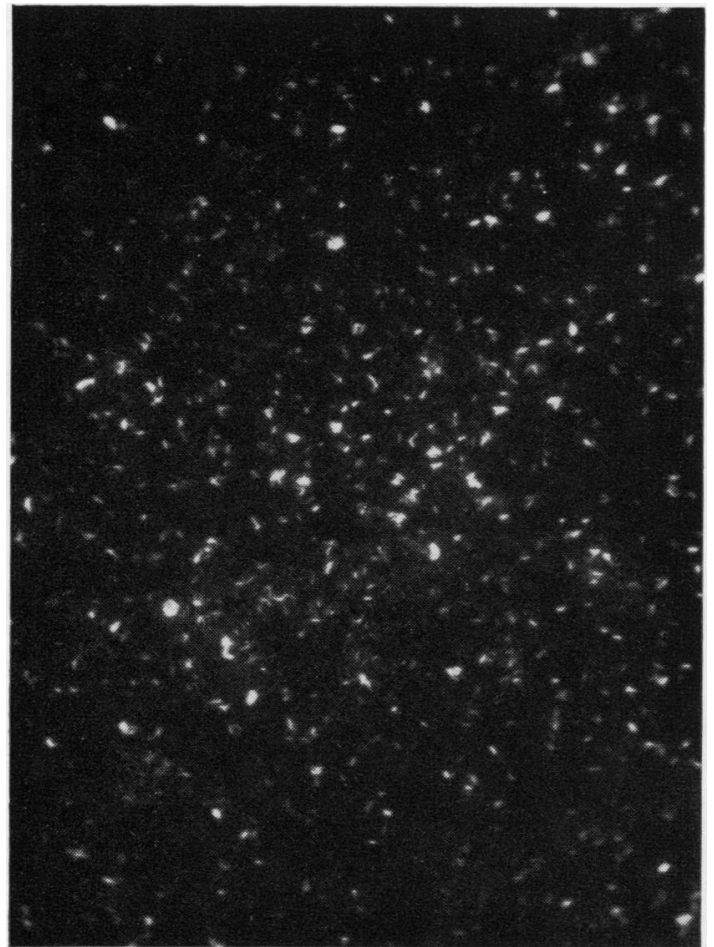

Fig. 1
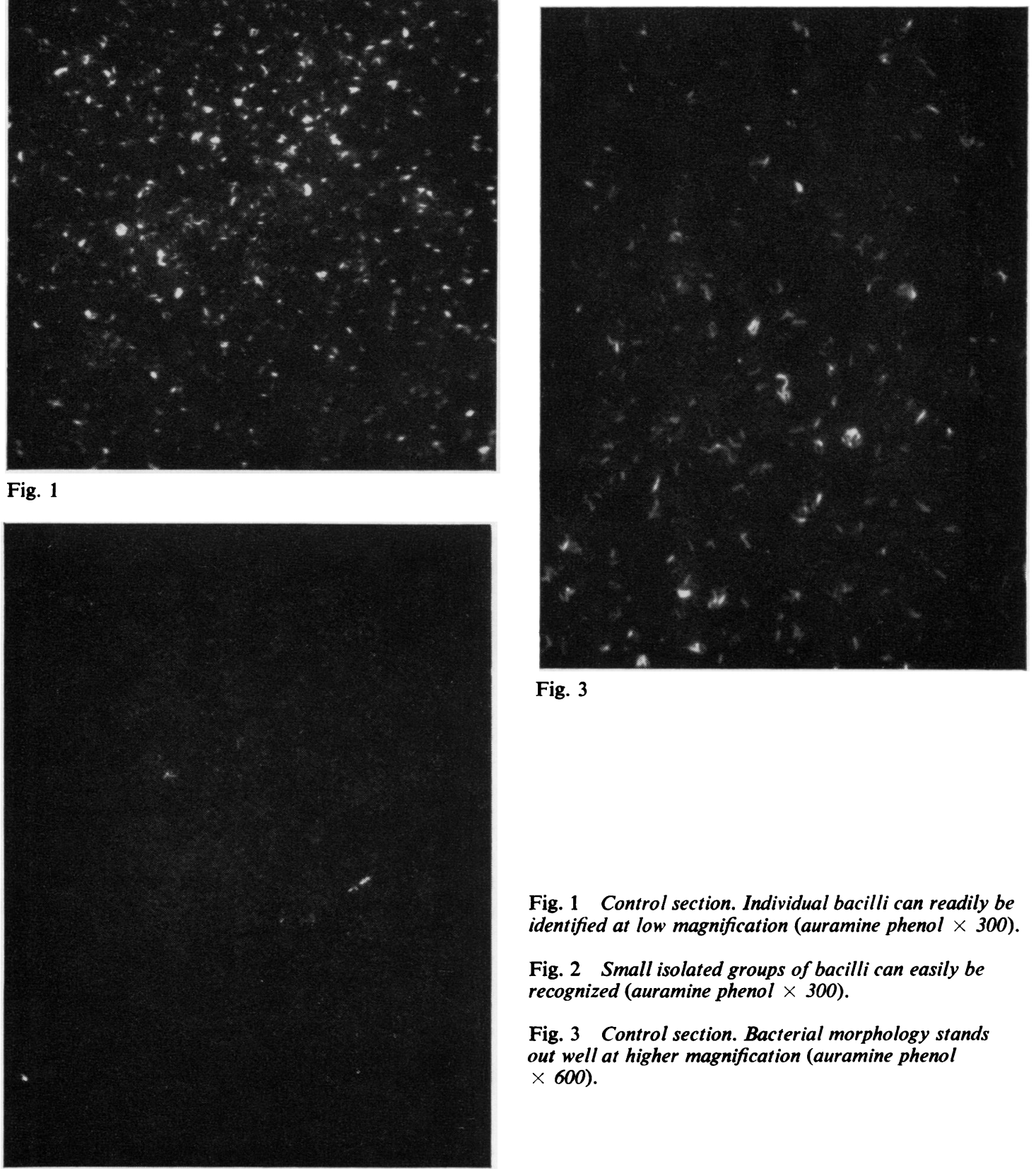

Fig. 3

Fig. 1 Control section. Individual bacilli can readily be identified at low magnification (auramine phenol $\times 300$ ).

Fig. 2 Small isolated groups of bacilli can easily be recognized (auramine phenol $\times 300$ ).

Fig. 3 Control section. Bacterial morphology stands out well at higher magnification (auramine phenol $\times 600$ ).

Fig. 2 


\begin{tabular}{|c|c|c|c|}
\hline \multirow[t]{2}{*}{ Technique } & \multicolumn{3}{|c|}{ Number of Positive Cases } \\
\hline & $\times 250$ Magnification & $\times 400$ Magnification & $\times 1000$ Magnification \\
\hline $\begin{array}{l}\text { Ziehl-Neelsen } \\
\text { Fite's } \\
\text { Armstrong Price } \\
\text { Auramine phenol }\end{array}$ & $\begin{array}{l}- \\
\overline{42}(60 \%)\end{array}$ & $\begin{array}{l}28(40 \%) \\
22(31 \cdot 4 \%) \\
19(27 \cdot 1 \%) \\
42(60 \%)\end{array}$ & $\begin{array}{l}33(47 \cdot 1 \%) \\
28(40 \%) \\
24(34 \cdot 3 \%) \\
42(60 \%)\end{array}$ \\
\hline
\end{tabular}

Table I Incidence of positive results for tubercle bacilli in 70 cases of histologically typical tuberculosis

\begin{tabular}{|c|c|c|c|}
\hline \multirow{2}{*}{ Technique } & \multirow{2}{*}{ Magnification } & \multicolumn{2}{|c|}{ Ziehl-Neelsen Positivity } \\
\hline & & $\begin{array}{l}\text { Positive Cases } \\
\text { Read as } \\
\text { Negative }\end{array}$ & $\begin{array}{l}\text { Negative Cases } \\
\text { Read as } \\
\text { Positive }\end{array}$ \\
\hline Fite's & $\begin{array}{l}400 \\
1000\end{array}$ & $\begin{array}{r}11 \\
5\end{array}$ & 1 \\
\hline Armstrong Price & $\begin{array}{l}400 \\
1000\end{array}$ & $\begin{array}{r}14 \\
8\end{array}$ & $\begin{array}{l}0 \\
0\end{array}$ \\
\hline Auramine phenol & $\begin{array}{l}400 \\
1000\end{array}$ & $\begin{array}{l}0 \\
0\end{array}$ & $\begin{array}{l}9 \\
9\end{array}$ \\
\hline
\end{tabular}

Table II Relationship between Ziehl-Neelsen positivity ( $\times 1000$ magnification) and results obtained by other techniques

\section{Results}

The results are shown in table I. In table II the results obtained with the alternative stains are compared with those of the Ziehl-Neelsen stain.

\section{CONTROL RESULTS}

Positive controls remained positive and negative controls remained negative throughout the investigation. The paraffin wax controls were also negative.

\section{Discussion}

Of the various fuchsin stains used in this investigation, the Ziehl-Neelsen method emerges as the best. It gives the highest yield of positive results, it is easy to interpret, and the staining technique is neither difficult nor prolonged. Neither Fite's method nor the Armstrong-Price modification were felt to be very reliable and both gave a high incidence of false negative results as compared with the ZiehlNeelsen stain; the staining technique for both is difficult and Fite's method requires overnight incubation.

All fuchsin methods for the detection of tubercle bacilli suffer, however, from the drawback that sections require prolonged and careful examination and our experience has been that a not inconsiderable proportion of cases will be missed using a $\times 400$ magnification and that most will be missed at a magnification of $\times 250$. With the fluorescent method, on the other hand, the slides can be fairly rapidly scanned at a magnification of $\times 250$ and the sections $\overrightarrow{\vec{\omega}}$ can thus be examined much more rapidly than with the fuchsin stains. Apart from this greater ease of examination, the yield of positive results is much greater with the fluorescent method than with the? other techniques and this has been confirmed in

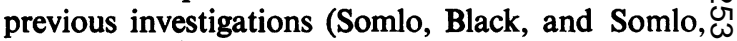
1969; Koch and Cote, 1965).

This raises the possibility that a proportion of $\rightarrow$ cases may have given a false positive reaction. As $>$ in all fluorescent methods, artefacts present a con-으․ stant problem, but as Mansfield (1970) points out, $\vec{\varphi}$ fluorescent staining techniques have been improved considerably in recent years, with the virtual elimina-: tion of tissue fluorescence, reducing the problem of artefacts to a minimum. In the present study, s bacterial morphology showed up clearly and only $\overline{0}$ morphologically definite bacilli were counted as positive. In the past, contamination of the paraffin $\cong$ wax by saprophytic, fluorescent-positive bacteria has $\overrightarrow{\overrightarrow{0}}$ probably given rise to some false positive results 3 (Wang, 1969), but the use of control sections of wax, in this investigation, excluded this possibility. Furthermore, of the nine cases in this study in which a positive fluorescent result was obtained on sections? which were Ziehl-Neelsen negative, one case was 3 positive by Fite's method; four were from cases in which other biopsies taken from nearby anatomical 윽 sites, at the same operation, were Ziehl-Neelsen positive and three were from biopsies from which a positive culture of tubercle bacilli was obtained. $\frac{7}{2}$ We would thus consider that these cases were unlikely to be false positives and this view is reinforced $N$ by the totally $>$ negative findings in the negative $N$ control sections. It should, perhaps, be stressed that ${ }^{N}$ the sections in which a positive result was obtained $\omega$ solely with the fluorescent method showed only a very few bacilli. The fact that none of the fluorescent- 0 negative cases were found to be positive by any $\mathbb{D}_{\overparen{D}}$ of the other methods suggests that it is a reliable technique and is unlikely to give false negative results.

The fluorescent auramine phenol technique would $\frac{\widehat{Q}}{\Phi}$ therefore appear to be the optimal method for the $\stackrel{\mathbb{Q}}{2}$ demonstration of tubercle bacilli in histologicalo material, its only drawback being the necessity 
of using a fluorescent microscope. It may be felt by some that this method has had insufficient testing for it to be used as the sole means of identifying tubercle bacilli. However, the fluorescent method, with its low incidence of false-negative results and its ease of performance, can be used as a screening test: fluorescent-negative sections can be reported as such, but positive sections can be confirmed by staining the same sections using the Ziehl-Neelsen method and concentrating on the area of the section shown to be positive by the fluorescent method, using, if necessary, the $\times 100$ oil immersion objective lens. This was, in fact, the means by which some of the sections, originally thought to be Ziehl-Neelsen negative, were found to be positive after our attention had been drawn by a positive fluorescent result.

We feel that there is a very strong case for the introduction of the auramine phenol fluorescent technique as a routine tool in diagnostic histopathology.

\section{References}

Armstrong, A. R., and Price, N. E. (1947). Acid-fast bacilli in paraffin sections of tuberculous tissue. Canad. med. Ass. J., 56, 83-84.

Fite, G. L. (1938). The staining of acid-fast bacilli in paraffin sections. Amer. J. Path., 14, 491-507.

Fite, G. L. (1940). The fuchsin-formaldehyde method of staining acid-fast bacilli in paraffin sections. J. Lab. clin. Med., 25. 743-744.

Koch, M. L., and Cote, R. A. (1965). Comparison of fluorescence microscopy with Ziehl-Neelsen stain for demonstration of acid-fast bacilli in smear preparations and tissue sections. Amer. Rev. resp. Dis., 91, 283-284.

Mansfield, |R. E. (1970). An improved method for the fluorochrome staining of mycobacteria in tissues and smears. Amer. J. clin. Path., 53, 394-406.

Somlo, A. M., Black, T. C., and Somlo, L. I. (1969). The value of fluorescence microscopy in the detection of acid-fast bacteria. Amer. J. clin. Path., 51, 519-522.

Wang, W. L. L. (1969). Contamination of tissue sections with acidfast bacilli as detected by fluorescence microscopy. Amer. $J$. clin. P.uth., 51, 71-75. 